\title{
Structural analysis of $8 / 6$ switched reluctance motor linear and non-linear models
}

\author{
J. D. González-San Román, J. U. Liceaga-Castro, I. I. Siller-Alcalá, E. Campero-Littlewood, \\ Universidad Autónoma Metropolitana-Azcapotzalco, \\ Av. San Pablo Xalpa 180, Reynosa Tamaulipas, Azcapotzalco, Ciudad de México, 02200 \\ México
}

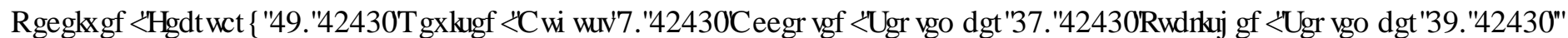

\begin{abstract}
This work presents the process of obtaining the simplified model of a switched reluctance motor (SRM) 8/6. Subsequently, the structure of the single-phase model is analyzed, obtaining an exact linearization and zero dynamics of the system. Finally, the model is linearized at an operating point set at $2000 \mathrm{rpm}$ The model includes Coulomb plus viscous friction nonlinearity and an ideal inverter circuit based on bridge converter topology. The simplified and linear models are simulated and compared in the Matlab ${ }^{\circledR} /$ Simulink software in order to validate the design of a classic controller using the linear model.
\end{abstract}

Keywords-. Switched reluctance motor (SRM), Simplified model, linear model, Exact I linearization, Zero dynamics.

\section{INTRODUCTION}

$\mathrm{I}$ $\mathrm{N}$ the last decades, the switched reluctance motor has attracted the attention of researchers, as a high-performance device in industrial applications, due to its advantages such as high torque at low speeds, large power-to-size ratio, efficient energy conversion, wide range of operating speed and easy cooling.[1]-[4] However, to properly take advantage of these characteristics of the SRM, optimal excitation and control of the same is necessary. This is not easy to obtain experimentally and it can even be a difficult and time-consuming task, depending on the complexity of the controllers to implement the necessary tests. For this reason, computational simulation of proper SRM models and its control systems becomes essential for the analysis and design of controllers for any SRM.

There are several ways to simulate an SRM, among which the most common are simulation using the Finite Element Method, such as the simulations developed in [5] and [6], where a good approximation is obtained to the dynamics of the real SRM, however this type of simulation can consume a lot of simulation time and computing power, for this reason, evaluating a controller, which may require a lot of tests, can be impractical. Another way to simulate an SRM is using lookup tables resulting from a finite element analysis as seen in [7] and [8], in this case the simulation becomes faster since most of the computation time is consumed by the analysis of previous finite element. And finally, the least used way to simulate an SRM is by programming the dynamic equations of the motor, as is usually done with other dc motors [9]-[11], which may belong to a non-linear model that considered the saturation of the material, or a simplified model that disregarded this phenomenon.

In this paper, the simplified non-linear model of an SRM 8/6 motor is obtained and later it is linearized at an operating point. The linearization of the model is carried out in order to analyze the structure of the motor and obtain a transfer function that, in future work, allows applying linear control techniques for the design of a classic controller. In addition, the model has an ideal inverter circuit that allows the motor to be operated in a similar way to a dc motor where, by reversing the polarity of the input voltage, the motor rotates in the opposite direction. Finally, different simulations are presented where the behavior of both models is compared in order to verify that it is feasible to design a controller based on the linear model.

This paper is organized as follows: section II shows the obtaining of the simplified non-linear model of the SRM. Section III shows the analysis of the structure of the singlephase non-linear model, where an exact linearization of the motor is obtained and subsequently its zero dynamics is found. Then, in section IV a linearization of the model is obtained at an operating point set at $2000 \mathrm{rpm}$, as well as its transfer function. In section $\mathrm{V}$ some general aspects for programming both models in Matlab/Simulink software are discussed. Section VI shows the simulation and comparison of both models in three different tests: first the behavior of the models in shutdown and demagnetization of the motor phases is observed, then a step type voltage input is applied and finally an input is applied sinusoidal type voltage. The discussion of 
results is presented in section VII and finally, section VIII shows the conclusions obtained from the work carried out.

\section{MATHEMATICAL MODEL OF THE SRM}

As in the modeling of other electromechanical machines, the starting point is the equations that govern the dynamics of the electrical sub-system (1) and the mechanical sub-system (2).

$$
\begin{gathered}
v_{j}=R i_{j}+\frac{d \psi_{j}}{d t} \\
\frac{\partial \omega}{\partial \tau}=\left(\tau_{e}-\tau_{l}-\tau_{f}\right) / J
\end{gathered}
$$

Where, $v_{j}, i_{j}, R$, and $\psi_{j}$ are the voltage, current, resistance and flow linkage of phase $j, \omega$ is the rotor's angular speed, $J$ is the moment of inertia, $\tau_{e}, \tau_{l}$ and $\tau_{f}$ are the corresponding electromagnetic, load and friction torque, the latter is represented by Coulomb plus viscous friction model [12] and [13], equation (3).

$$
\tau_{f}=D \omega+\Delta \operatorname{sgn}(\omega)
$$

Where, $D$ is the viscous coefficient and $\Delta$ is the Coulomb friction force. The model that is developed here is called the simplified model and considers the following [14]: here is no saturation of the material, therefore, the flux linkage are described by the product of the inductance and the phase current, the fringing effects are neglected, the mutual inductance is negligible, the motor phases are identical and the inductance is a function of the rotor position [15] given by (4).

$$
L_{j}=L_{0}-L_{1} \cos \left(N_{r} \theta-(j-1) 2 \pi / N\right)
$$

Where, $\theta$ is the position of the rotor, $L_{0}$ is the self-inductance of each phase, $L_{1}$ is the inductance dependent on the position of the rotor, $N_{r}$ is the number of rotor poles and $N$ is the number of phases. Substituting (4) in (1), we obtain the equation that represents the electrical subsystem (5).

$$
\frac{d i_{j}}{d t}=\frac{v_{j}-R i_{j}-L_{1} N_{r} i_{j} \omega \sin \left(N_{r} \theta-(j-1) 2 \pi / N\right)}{L_{0}-L_{1} \cos \left(N_{r} \theta-(j-1) 2 \pi / N\right)}
$$

The equation for the mechanical subsystem is obtained by the co-energy method, therefore, the torque generated by each phase is given by (6)

$$
\tau_{j}\left(\theta, i_{j}\right)=\frac{\partial W_{j}^{\prime}}{\partial \theta}
$$

The co-energy function is given by (7)

$$
W_{j}^{\prime}\left(\theta, i_{j}\right)=\int_{0}^{i_{j}} \psi_{j}\left(\theta, i_{j}\right) d i_{j}
$$

Solving (6) and (7), the torque of each phase is obtained. Finally, the electromagnetic torque is the sum of the individual torques, therefore, the mechanical subsystem is now described by (8)

$$
\frac{d \omega}{d t}=\frac{1}{J}\left[\frac{N_{r} L_{1}}{2} \sum_{j=1}^{N} i_{j}^{2} \sin \left(N_{r} \theta-(j-1) \frac{2 \pi}{N}\right)-\tau_{l}-\tau_{f}\right]
$$

\section{STRUCTURE ANALYSIS OF THE NONLINEAR MODEL}

To properly design the controller of any system, it is essential to know the latter thoroughly. For this reason, it is necessary to analyze the structure of the non-linear model and subsequently the linear model, since both models are one of the main sources of information about the motor. In order to know in depth, the non-linear model of the motor, its exact linearization was carried out with which, the degree and relative degree of it was known, and a linear and controllable form was found that can be useful in subsequent studies that seek to employ nonlinear control techniques.

From the simplified model obtained above, it can be noted that the complete model of an SRM 8/6 consists of eight equations in total, where there are four voltage inputs, one for load torque, and two outputs, one for torque and one for speed. Therefore, an SRM 8/6 is a multiple input multiple output system (MIMO), the structural analysis of which becomes extremely extensive and complex. On the other hand, if it is considered that each of the phases are activated sequentially and independently, the load torque is a disturbance of the system and remembering that the phases are identical, it is possible to analyze a single motor phase, where there is only one voltage input and one speed output, that is, a system one input one output (SISO).

\section{A. Exact linearization}

To analyze the system, it is necessary to rewrite it in the state space, whose form is shown in (9).

$$
\begin{gathered}
\dot{x}=f(x)+g(x) u \\
y=h(x)
\end{gathered}
$$
(10)

To pass the model to the state space, the following is defined

$$
\begin{aligned}
& x_{1}=: i \\
& x_{2}=: \theta \\
& x_{3}=: \omega
\end{aligned}
$$

The single-phase model in state space is shown in (11). 


$$
\begin{aligned}
& \left(\begin{array}{c}
\dot{x}_{1} \\
\dot{x}_{2} \\
\dot{x}_{3}
\end{array}\right)=\left(\begin{array}{c}
-\frac{x_{1} R+L_{1} N_{r} x_{1} x_{3} \sin \left(N_{r} x_{2}\right)}{L_{0}-L_{1} \cos \left(N_{r} x_{2}\right)} \\
x_{3} \\
\frac{N_{r} L_{1} x_{1}^{2}}{2 J} \sin \left(N_{r} x_{2}\right)-\frac{D x_{3}+\Delta \operatorname{sgn}\left(x_{3}\right)}{J}
\end{array}\right) \\
& +\left(\begin{array}{c}
\frac{1}{L_{0}-L_{1} \cos \left(N_{r} x_{2}\right)} \\
0 \\
0
\end{array}\right) u \\
& y=x_{3}
\end{aligned}
$$

To start the exact linearization, it must be verified that the system is transformable to a linear and controllable one, which is possible if and only if the following conditions are met [16]:

- The matrix $\left[g\left(x_{0}\right), a d_{f} g\left(x_{0}\right), \ldots, a d_{f}^{n-l} g\left(x_{0}\right)\right]$ has rank $\mathrm{n}$.

- The distribution $D=\left\{g, a d_{f} g, \ldots, a d_{f}^{n-2} g\right\}$ is involutive near $x_{0}$

Now we proceed to calculate each of the elements of the matrix and the distribution, as seen from (12) to (14)

$$
\frac{\partial g}{\partial x}=\left(\begin{array}{ccc}
0 & -\frac{L_{1} N_{r} \sin \left(N_{r} x_{2}\right)}{\left(L_{0}-L_{1} \cos \left(N_{r} x_{2}\right)\right)^{2}} & 0 \\
0 & 0 & 0 \\
0 & 0 & 0
\end{array}\right)
$$

$$
\operatorname{ad}_{f} g(x)=\left(\begin{array}{c}
\frac{R}{\left(L_{0}-L_{1} \cos \right)^{2}} \\
0 \\
-\frac{L_{1} N_{r} x_{1} \sin \left(N_{r} x_{2}\right)}{J\left(L_{0}-L_{1} \cos \left(N_{r} x_{2}\right)\right)}
\end{array}\right)
$$

$$
a d_{f}^{2} g(x)=\left(\begin{array}{c}
\frac{J R\left[R-L_{1} N_{r} x_{3} \sin \left(N_{r} x_{2}\right)\right.}{J\left(L_{0}-L_{1} \cos \left(N_{r} x_{2}\right)\right)^{3}} \\
\frac{L_{1} N_{r} x_{1} \sin \left(N_{r} x_{2}\right)}{J\left(L_{0}-L_{1} \cos \left(N_{r} x_{2}\right)\right)} \\
\frac{J\left[L_{1}^{2} N_{r}^{2} x_{1} x_{3} \sin ^{2}\left(N_{r} x_{2}\right)-L_{1} L_{0} N_{r}^{2} x_{1} x_{3} \cos \left(N_{r} x_{2}\right)\right.}{\left[J\left(L_{0}-L_{1} \cos \left(N_{r} x_{2}\right)\right)\right]^{2}}
\end{array}\right.
$$

$$
\left.\begin{array}{c}
\frac{\left.-\left[L_{0}-L_{1} \cos \left(N_{r} x_{2}\right)\right] L_{1}^{2} N_{r}^{2} x_{1}^{2} \sin ^{2}\left(N_{r} x_{2}\right)\right]}{J\left(L_{0}-L_{1} \cos \left(N_{r} x_{2}\right)\right)^{3}} \\
0 \\
\ldots \\
\frac{\left.+L_{1} N_{r} x_{1} R \sin \left(N_{r} x_{2}\right)+L_{1}^{2} N_{r}^{2} x_{1} x_{3}\right]}{\left[J\left(L_{0}-L_{1} \cos \left(N_{r} x_{2}\right)\right]^{2}\right.} \\
0 \\
-\frac{\left[L_{0}-L_{1} \cos \left(N_{r} x_{2}\right)\right] D N_{r} L_{1} x_{1} \sin \left(N_{r} x_{2}\right)}{\left[J\left(L_{0}-L_{1} \cos \left(N_{r} x_{2}\right)\right)\right]^{2}}
\end{array}\right)
$$

It is verified that when evaluating (12) to (14) at $x_{0}$, the matrix maintains rank $n$ equal to 3 . Next, it is observed that the distribution $D$ has rank 2, therefore, it only remains to verify that the rank of the matrix $\left(g, a d_{f} g,\left[g, a d_{f} \mathrm{~g}\right]\right)$ is the same that of the distribution D. Equation (15) shows the result of the operation $\left[g, a d_{f} g\right]$.

$$
\left[g, a d_{f} g\right]=\left(\begin{array}{c}
0 \\
0 \\
-\frac{L_{1} N_{r} \sin \left(N_{r} x_{2}\right)}{J\left(L_{0}-L_{1} \cos \left(N_{r} x_{2}\right)\right)^{2}}
\end{array}\right)
$$

With the help of (15) it can easily be verified that the matrix $\left(g, a d_{f} g,\left[g, a d_{f} g\right]\right)$ has rank 2 and, therefore, the distribution $D$ is involutive, which shows that the system is transformable to a linear and controllable system. Now the relative degree $r$ of the system is calculated to determine if it is necessary to obtain an output function different from the original one. The system is said to have relative degree $r$ at point $x_{0}$ if the following is satisfied [16]:

- $\quad L_{g} L_{f}^{k} h(x)=0$ for all $x$ in a neighborhood of $x_{0}$ and all $k$ $<r-1$.

- $L_{g} L_{f}^{r-1} h(x) \neq 0$.

In (16) are shown the corresponding operations.

$$
\begin{gathered}
L_{g} h(x)=0 ; \\
L_{g} L_{f} h(x)=\frac{L_{1} N_{r} x_{1} \sin \left(N_{r} x_{2}\right)}{J\left(L_{0}-L_{1} \cos N_{r} x_{2}\right)} \neq 0 ;
\end{gathered}
$$

From (16) it follows that the relative degree of the system is equal to 2 for all $x_{1} \neq 0$ and $x_{2} \neq k \pi$ with $k \in \mathbb{Z}$. Since $r \neq n$, it is necessary to look for an output function $\lambda$ such that $d \lambda(x)(g(x)$, $\left.a d_{f} g(x), \ldots, a d^{n-2} f(x)\right)=0$, whose development is shown in (17). 


$$
\begin{aligned}
& \left(\frac{1}{L_{0}-L_{1} \cos \left(N_{r} x_{2}\right)} \frac{\partial \lambda}{\partial x_{1}}, \frac{R}{\left(L_{0}-L_{1} \cos \left(N_{r} x_{2}\right)\right)^{2}} \frac{\partial \lambda}{\partial x_{1}}\right. \\
& \left.-\frac{L_{1} N_{r} x_{1} \sin \left(N_{r} x_{2}\right)}{J\left(L_{0}-L_{1} \cos \left(N_{r} x_{2}\right)\right)} \frac{\partial \lambda}{\partial x_{3}}\right)
\end{aligned}
$$

From (17) it can be seen that it is feasible to choose $x_{2}$ as the output function. Again, the relative degree is calculated to verify that it coincides with the degree of the system, the operations necessary for this task are shown in (18).

$$
\begin{gathered}
L_{g} \lambda(x)=0 \\
L_{g} L_{f} \lambda(x)=0 \\
L_{g} L_{f}^{2} \lambda(x)=\frac{L_{1} N_{r} x_{1} \sin \left(N_{r} x_{2}\right)}{J\left(L_{0}-L_{1} \cos \left(N_{r} x_{2}\right)\right)} \neq 0
\end{gathered}
$$

From (18) it is obtained that the relative degree is equal to 3 for all $x_{1} \neq 0$ and $x_{2} \neq k$ with $k \in \mathbb{Z}$ as it happened in the previous calculation. It is from this point that it is possible to perform the linearization via state feedback, for which, the input $u$ must be obtained through (19).

$$
u=\frac{-L_{f}^{3} \lambda(x)+v}{L_{g} L_{f}^{2} \lambda(x)}
$$
(20).

Where $L_{g} L_{f}^{2} \lambda(x)$ is taken from (18) and $L_{f}^{3} \lambda(x)$ is shown in

$$
\begin{aligned}
& L_{f}^{3} \lambda(x)=\left\{L_{0} L_{1} N_{r}^{2} x_{1}^{2} x_{3} \cos \left(N_{r} x_{2}\right)\right. \\
& -2 L_{1} N_{r} R x_{1}^{2} \sin \left(N_{r} x_{2}\right) \\
& -L_{1}^{2} N_{r}^{2} x_{1}^{2} x_{3}\left(1+\sin ^{2}\left(N_{r} x_{2}\right)\right)-\frac{2 D}{J}\left[L_{0}\right. \\
& \left.-L_{1} \cos \left(N_{r} x_{2}\right)\right]\left[L_{1} N_{r} x_{1}^{2} \sin \left(N_{r} x_{2}\right)\right. \\
& \left.\left.-D x_{3}-\Delta \operatorname{sgn}\left(x_{3}\right)\right]\right\} / 2 J\left[L_{0}-L_{1} \cos \left(N_{r} x_{2}\right)\right]
\end{aligned}
$$

The input $u$ obtained is shown in (21).

$$
\begin{aligned}
& u=\left\{-L_{0} L_{1} N_{r}^{2} x_{1}^{2} x_{3} \cos \left(N_{r} x_{2}\right)\right. \\
& +2 L_{1} N_{r} R x_{1}^{2} \sin \left(N_{r} x_{2}\right) \\
& +L_{1}^{2} N_{r}^{2} x_{1}^{2} x_{3}\left(1+\sin ^{2}\left(N_{r} x_{2}\right)\right)+\frac{2 D}{J}\left[L_{0}\right. \\
& \left.-L_{1} \cos \left(N_{r} x_{2}\right)\right]\left[L_{1} N_{r} x_{1}^{2} \sin \left(N_{r} x_{2}\right)\right. \\
& \left.\left.-D x_{3}-\Delta \operatorname{sgn}\left(x_{3}\right)\right]+v\right\} / L_{1} N_{r} x_{1} \sin \left(N_{r} x_{2}\right)
\end{aligned}
$$

Finally, the coordinates transformation is shown in (22).

$$
\begin{gathered}
z_{1}=\lambda(x)=x_{2} \\
z_{2}=L_{f} \lambda(x)=x_{3} \\
z_{3}=L_{f}^{2} \lambda(x)=\frac{N_{r} L_{1}}{2 J} x_{1}^{2} \sin \left(N_{r} x_{2}\right) \\
-\frac{1}{J}\left[D x_{3}+\Delta \operatorname{sgn}\left(x_{3}\right)\right]
\end{gathered}
$$

\section{B. Zero dynamics}

In the analysis of non-linear systems, it is very useful to know the zero dynamics, which has a similar role to the zeros in linear systems. In this case, zero dynamics is the set of all states $x_{0}$ and inputs $u\left(x_{0}\right)$, defined for all $t$ in the neighborhood of $t=0$ that make the output identical to zero. The procedure to find the zero dynamics of any system consists of zeroing all the states $z_{1}, z_{2}$, $\ldots, z_{r-1}$, and $z_{\mathrm{r}}$ and substituting it in the dynamics of the system. To perform these steps, it is necessary to find a coordinate transformation, such as the one obtained in the exact linearization, but in this case respecting the output function $h(x)$, for this we have the following (23).

$$
\begin{gathered}
z_{1}=\phi_{1}(x)=h(x)=x_{3} \\
z_{2}=\phi_{2}(x)=L_{f} h(x)=\frac{N_{r} L_{1}}{2 J} x_{1}^{2} \sin \left(N_{r} x_{2}\right) \\
-\frac{1}{J}\left[D x_{3}+\Delta \operatorname{sgn}\left(x_{3}\right)\right] \\
z_{3}=\phi_{3}(x)
\end{gathered}
$$

We are looking for a function $\phi_{3}(x)$ such that $L_{g} \phi_{3}(x)=0$, it is proposed that $\phi_{3}(x)=x_{2}$, now it is verified that the Jacobian of $\Phi$ is not a singular matrix, this is easy to do through its determinant (24).

$$
\operatorname{det}\left(\frac{\partial \Phi}{\partial x}\right)=\frac{N_{r} L_{1}}{J} x_{1} \sin \left(N_{r} x_{2}\right)
$$

The inverse transformation is given by (25).

$$
\begin{gathered}
x_{3}=z_{1} \\
x_{2}=z_{3} \\
x_{1}=\sqrt{\frac{2\left[J z_{2}+D z_{1}+\Delta \operatorname{sgn}\left(z_{1}\right)\right]}{N_{r} L_{1} \sin \left(N_{r} z_{3}\right)}}
\end{gathered}
$$

With the coordinate transformation, the dynamics of the system is described by (26). 


$$
\begin{gathered}
\dot{z}_{1}=z_{2} \\
\dot{z}_{2}=b(z)+a(z) u \\
\dot{z}_{3}=q_{3}(z)
\end{gathered}
$$

Where:

$$
\begin{gathered}
a(z)=L_{g} L_{f} h\left(\Phi^{-1}(z)\right) \\
b(z)=L_{f}^{2} h\left(\Phi^{-1}(z)\right) \\
q_{3}(z)=L_{f} \phi_{3}\left(\Phi^{-1}(z)\right)
\end{gathered}
$$

From (16) and (25), you get $a(z)$ shown in (28).

$$
\begin{aligned}
& a(z)=\left\{2 N _ { r } L _ { 1 } \operatorname { s i n } ( N _ { r } z _ { 3 } ) \left[J z_{2}+D z_{1}\right.\right. \\
& \left.\left.+\Delta \operatorname{sgn}\left(z_{1}\right)\right]\right\} / J\left[L_{0}-L_{1} \cos \left(N_{r} z_{3}\right)\right]
\end{aligned}
$$

Calculate $L_{f}^{2} h(x)(29)$

$L_{f}^{2} h(x)=-\frac{N_{r} L_{1} x_{1}^{2} \sin \left(N_{r} x_{2}\right)\left[R+L_{1} N_{r} x_{3} \sin \left(N_{r} x_{2}\right)\right]}{L_{0}-L_{1} \cos \left(N_{r} x_{2}\right)}$

$+\frac{N_{r}^{2} L_{1}}{2 J} x_{1}^{2} x_{3} \cos \left(N_{r} x_{2}\right)-\frac{D}{J^{2}}\left[\frac{N_{r} L_{1}}{2} x_{1}^{2} \sin \left(N_{r} x_{2}\right)\right.$

$\left.-D x_{3}-\Delta \operatorname{sgn}\left(x_{3}\right)\right]$

From (25) and (29) is found b (z) as shown in (30)

$$
\begin{aligned}
& b(z)=\left\{-2\left[J z_{2}+D z_{1}+\Delta \operatorname{sgn}\left(z_{1}\right)\right][R+\right. \\
& \left.\left.L_{1} N_{r} z_{1} \sin \left(N_{r} z_{3}\right)\right]\right\} / J\left[L_{0}-L_{1} \cos \left(N_{r} z_{3}\right)\right]+ \\
& \frac{N_{r} z_{1}\left[J z_{2}+D z_{1}+\Delta \operatorname{sgn}\left(z_{1}\right)\right] \cos \left(N_{r} z_{3}\right)}{J \sin \left(N_{r} z_{3}\right)}-\frac{D}{J} z_{2}
\end{aligned}
$$

To find $\mathrm{q}_{3}(\mathrm{z})$ we have the following (31)

$$
\begin{gathered}
\frac{\partial \phi_{3}}{\partial x}=(0,1, \quad 0) ; \\
L_{f} \phi_{3}(x)=x_{3} ; \\
q_{3}(z)=L_{f} \phi_{3}\left(\Phi^{-1}(z)\right)=z_{1} ;
\end{gathered}
$$

We make $z_{1}=z_{2}=0$ and substitute in (28) and (30), obtaining (32) and (33) correspondingly.

$$
\begin{gathered}
\left.a(z)\right|_{z_{1}=0, z_{2}=0}=\frac{2 N_{r} L_{1} \sin \left(N_{r} z_{3}\right)\left[\Delta \operatorname{sgn}\left(x_{3}\right)\right]}{J\left[L_{0}-L_{1} \cos \left(N_{r} z_{3}\right)\right]} \\
\left.b(z)\right|_{z_{1}=0, z_{2}=0}=-\frac{2 R\left[\Delta \operatorname{sgn}\left(x_{3}\right)\right]}{J\left[L_{0}-L_{1} \cos \left(N_{r} z_{3}\right)\right]}
\end{gathered}
$$

The input $u$ is defined by (34).

$$
u=-\left.\frac{b(z)}{a(z)}\right|_{z_{1}=0, z_{2}=0}=\frac{R}{N_{r} L_{1} \sin \left(N_{r} z_{3}\right)}
$$

Since no dynamic equation of the system was different to zero, it is said that the system does not have zero dynamics.

\section{Model LINEARIZATION}

To obtain the linearization of the model, it was considered that the inductance of the phase has a constant value, which is obtained by considering a constant value of the rotor position $\theta=\Theta$. Regarding the speed equation, only the positive part is considered, that is, when $\omega>0$, because the operating point is at 2000rpm. The result of the previous considerations is the set of equations to linearize are those shown in (35).

$$
\begin{gathered}
\frac{d i}{d t}=-\frac{R}{L_{0}-L_{1} \cos \left(N_{r} \theta\right)} i-\frac{L_{1} N_{r} \sin \left(N_{r} \theta\right)}{L_{0}-L_{1} \cos \left(N_{r} \theta\right)} i \omega \\
+\frac{1}{L_{0}-L_{1} \cos \left(N_{r} \theta\right)} v \\
\frac{d \omega}{d t}=\frac{L_{1} N_{r} \sin \left(N_{r} \theta\right)}{2 J} i^{2}-\frac{D}{J} \omega-\frac{\Delta}{J}-\frac{1}{J} \tau_{l}
\end{gathered}
$$

To simplify the model and pass it to the state space, the following is defined (36).

$$
\begin{array}{cc}
a_{1}=: \frac{R}{L_{0}-L_{1} \cos \left(N_{r} \Theta\right)} ; & b_{1}=: \frac{L_{1} N_{r} \sin \left(N_{r} \Theta\right)}{2 J} ; \\
a_{2}=: \frac{L_{1} N_{r} \sin \left(N_{r} \Theta\right)}{L_{0}-L_{1} \cos \left(N_{r} \Theta\right)} ; & b_{2}=: D / J ; \\
a_{3}=: \frac{1}{L_{0}-L_{1} \cos \left(N_{r} \Theta\right)} ; & b_{3}=: \Delta / J ; \\
x_{1}=: i ; & b_{4}=: 1 / J ; \\
x_{2}=: \omega ;
\end{array}
$$

Therefore, the model in the state space is defined by (37) and written in a matrix form, it is as shown in (38). 


$$
\begin{gathered}
\dot{x}_{1}=-a_{1} x_{1}-a_{2} x_{1} x_{2}+a_{3} v \\
\dot{x}_{2}=b_{1} x_{1}^{2}-b_{2} x_{2}-b_{3}-b_{4} \tau_{l} \\
\dot{x}=\left(\begin{array}{c}
\dot{x}_{1} \\
\dot{x}_{2}
\end{array}\right)=\left(\begin{array}{c}
-a_{1} x_{1}-a_{2} x_{1} x_{2}+a_{3} v \\
b_{1} x_{1}^{2}-b_{2} x_{2}-b_{3}-b_{4} \tau_{l}
\end{array}\right)=f(x, u) ; \\
y=x_{2}=h(x) ;
\end{gathered}
$$

Now we find a parameterized operating point for $x_{2}=x_{2}{ }^{0}$, from which we obtain (39).

$$
x_{1}^{0}=\sqrt{\frac{b_{2} x_{2}^{0}+b_{3}+b_{4} \tau_{l}}{b_{1}}} ; V=\frac{x_{1}^{0}\left(a_{1}+a_{2} x_{2}^{0}\right)}{a_{3}} ;
$$

The matrix form, which we want to get to, is (40)

$$
\begin{gathered}
\dot{x}=A x+B u \\
y=C x
\end{gathered}
$$

When calculating each of the Jacobian matrices, we obtain (41).

$$
\begin{aligned}
& A=\left.\frac{\partial f(x, u)}{\partial x}\right|_{x_{1}^{0}, x_{2}^{0}}=\left(\begin{array}{cc}
-\left(a_{1}+a_{2} x_{2}^{0}\right) & -a_{2} x_{1}^{0} \\
2 b_{1} x_{1}^{0} & -b_{2}
\end{array}\right) ; \\
& B=\left.\frac{\partial f(x, u)}{\partial u}\right|_{x_{1}^{0}, x_{2}^{0}}=\left(\begin{array}{cc}
a_{3} & 0 \\
0 & -b_{4}
\end{array}\right) ; \\
& C=\left.\frac{\partial h(x)}{\partial x}\right|_{x_{1}^{0}, x_{2}^{0}}=\left(\begin{array}{ll}
0 & 1
\end{array}\right) ;
\end{aligned}
$$

Where the input vector is (42)

$$
u=\left(\begin{array}{ll}
v & \tau_{l}
\end{array}\right)^{T}
$$

Assuming that the input torque is equal to zero, $\tau_{l}=0$, the operating point and input matrix $B$ are recalculated (43).

$$
\begin{gathered}
x_{1}^{0}=\sqrt{\frac{b_{2} x_{2}^{0}+b_{3}}{b_{1}}} ; V=\frac{x_{1}^{0}\left(a_{1}+a_{2} x_{2}^{0}\right)}{a_{3}} ; \\
B=\left(\begin{array}{c}
a_{3} \\
0
\end{array}\right) ;
\end{gathered}
$$

Then the transfer function is calculated by (44), the calculation progress is shown from (45) to (47).

$$
\begin{gathered}
G(s)=\frac{C \operatorname{Adj}(s I-A)^{T} B}{\operatorname{det}(s I-A)} \\
\operatorname{Adj}(s I-A)^{T}=\left(\begin{array}{cc}
s+b_{2} & -a_{2} x_{1}^{0} \\
2 b_{1} x_{1}^{0} & s+\left(a_{1}+a_{2} x_{2}^{0}\right)
\end{array}\right) \\
\operatorname{det}(s I-A)=s^{2}+\left(a_{1}+a_{2} x_{2}^{0}+b_{2}\right) s \\
+b_{2}\left(a_{1}+a_{2} x_{2}^{0}\right)+2 a_{2} b_{1}\left(x_{1}^{0}\right)^{2} \\
G(s)=2 a_{3} b_{1} x_{1}^{0} /\left[s^{2}+\left(a_{1}+a_{2} x_{2}^{0}+b_{2}\right) s\right. \\
\left.+b_{2}\left(a_{1}+a_{2} x_{2}^{0}\right)+2 a_{2} b_{1}\left(x_{1}^{0}\right)^{2}\right]
\end{gathered}
$$

Finally, substituting in (47) the parameters of the SRM RA130135 from System Tech, Fig. 1, with eight poles on the stator, six poles on the rotor (8/6) and four phases, given by:

- $\operatorname{Vmax}=24 \mathrm{Vdc}$

- $N=4$

- $N_{r}=6$

- $J=3.9063 \mathrm{Kg} \cdot \mathrm{m}^{2}$

- $\tau_{l}=0.01 \mathrm{~N} \cdot \mathrm{m}$

- $D=0.0001 \mathrm{~N} \cdot \mathrm{m} / \mathrm{rad} / \mathrm{s}$

- $\Delta=0.005 \mathrm{~N} \cdot \mathrm{m}$

- $R=1 \Omega$

- $L_{0}=2.1 \mathrm{mH}$

- $L_{1}=1.3 \mathrm{mH}$

- $\Theta=2^{\circ}$

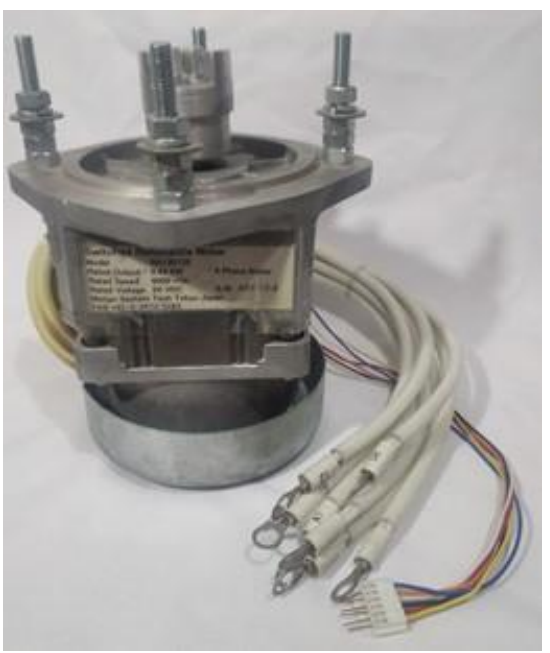

Fig. 1. Switched reluctance motor.

the single-phase transfer function of the switched reluctance motor is obtained, shown in (22). This transfer function has no zeros and has a pair of widely separated poles. Therefore, the transfer function obtained coincides with what is normally obtained in direct current motors, in which there is a dominant pole from the mechanical sub-system and a non-dominant pole 
from the electrical sub-system, as corroborated in jobs like [17], [18], and [19].

$$
\begin{gathered}
G(s)=\frac{283470}{s^{2}+1619.7 s+6740.2} ; \\
\text { poles }=\{-4.2,-1615.5\}
\end{gathered}
$$

\section{MODEL PROGRAMMING}

Programming the motor model is simple thanks to the tools provided by Simulink in particular the Matlab function block, in which equations of the model are directly entered and through integrator blocks the variables are obtained and divided into six Matlab function blocks, four to the motor and two corresponding to the inverter circuit. The motor blocks were programmed using the equations obtained in the mathematical model section according to the following list:

- Block of inductances, equation (4).

- Electrical system block, equation (5).

- Mechanical system block, equation (6).

- Speed block, equation (2).

On the other hand, the inverter circuit that was programmed is based on the ideal classical converter or bridge converter topology [20], that is, the physical limitations of the transistors are not taken into account, only the logic in which they operate. Commonly, any inverter circuit implements some current regulation technique as a protection for the motor, since the resistance of the phases is usually relatively small causing a current demand that can damage the motor. In order to program this circuit, it was divided into two blocks: the first one handles the switching logic of the phases and the other regulates the current of the phases.

For the switching logic block, it is considered that, according to (8), the sign of the torque is independent of the sign of the current, and is only affected by the sinusoidal function, which corresponds to the derivative of inductance. That is, the torque produced by each phase will be positive whenever the inductance increases and negative when the inductance decreases. From the above, the activation sequences of the phases for clockwise and counter-clockwise rotation are deduced. The current regulation block implements the hysteresis technique [3] since it directly regulates the current by turning the phase on and off, keeping the current within a hysteresis window defined by a minimum and maximum current value, both blocks of the inverter circuit are programmed in the Matlab function.

The process described above was applied to the programming of the non-linear model of the motor, in the case of the linear model, only the inductance block is omitted and the equations of the blocks of the electrical and mechanical systems are exchanged for their linear counterpart.

\section{SIMULATION AND COMPARISON OF THE MODELS}

\section{A. Simulation with phases in shutdown and demagnetization}

The first experiment that is presented consists of simulating both models at the operating point of 2000rpm with phase shutdown, that is, without demagnetization and with lower and upper current limits of 6 and 7A. The test lasts 3 seconds in which the speed and torque responses of the models reach a stable state, as shown in Fig. 2 and 3 respectively.

For the next test, the simulation is repeated, but now demagnetization is applied to the phases, again the speed and torque responses are observed, as shown in Fig. 4 and 5. Additionally, Fig. 6 and 7 show the current responses of phase A in steady state.

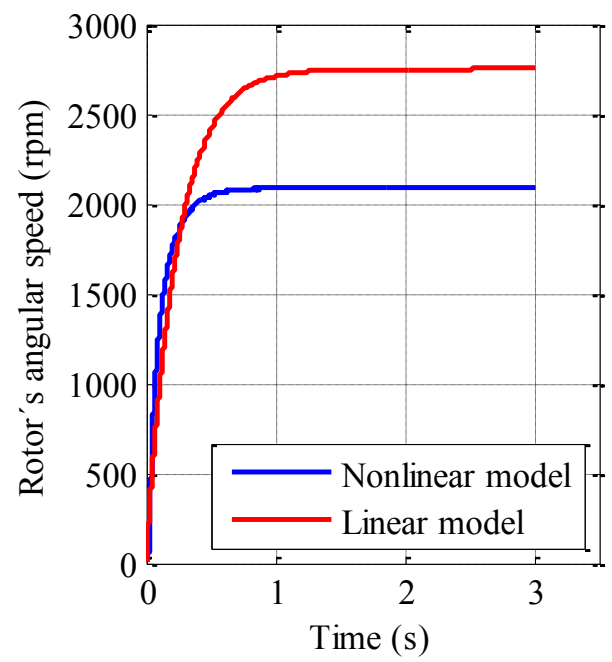

Fig. 2. Rotor's angular speed response, without demagnetization.

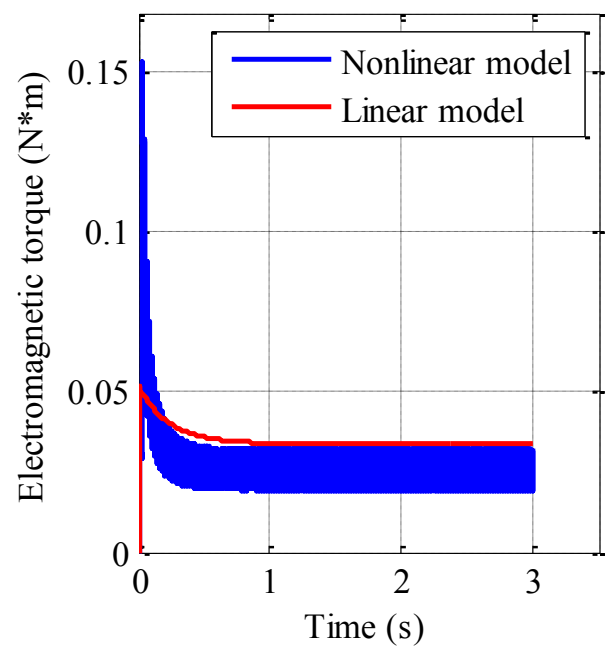

Fig. 3. Electromagnetic torque response, without demagnetization. 


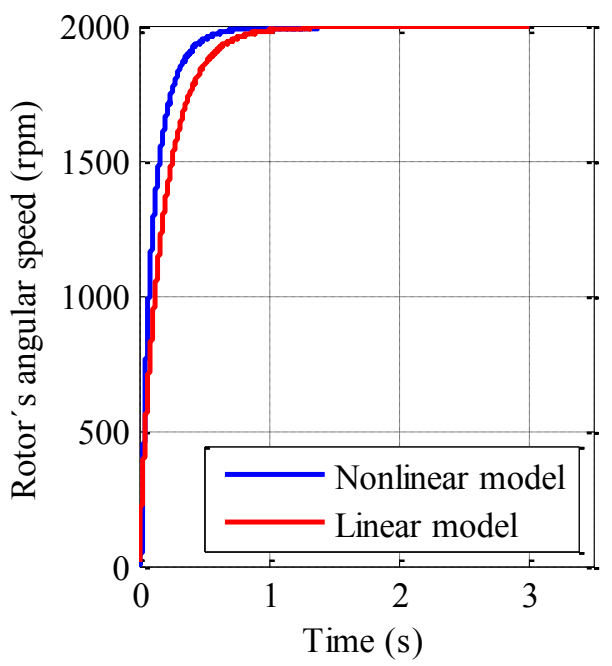

Fig. 4. Rotor's angular speed response, with demagnetization.

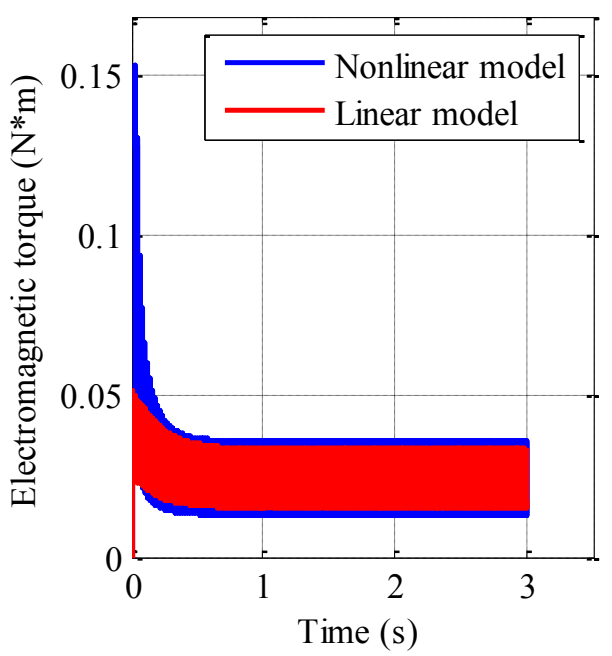

Fig. 5. Electromagnetic torque response, with demagnetization.

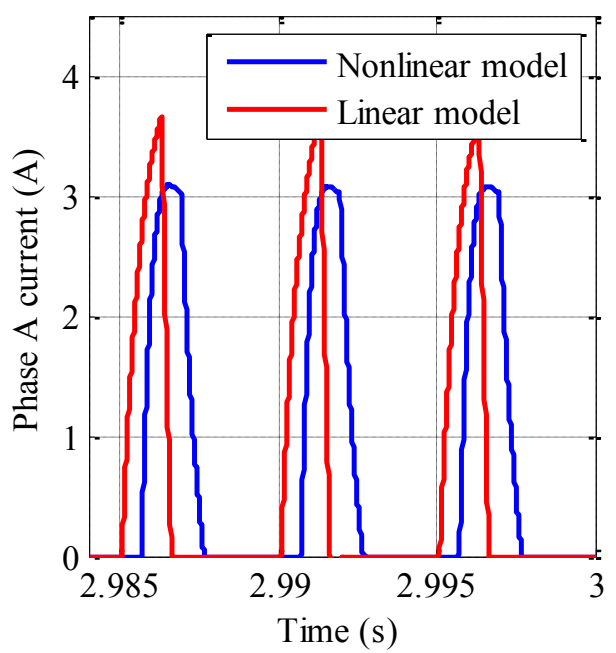

Fig. 6. Phase A current response, with demagnetization.

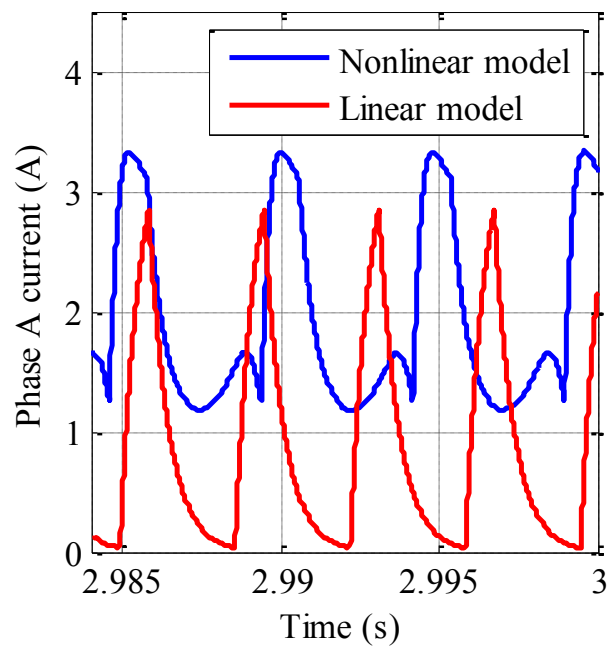

Fig. 7. Phase A current response, without demagnetization.

\section{B. Step input simulation}

For this experiment, the behavior of both models of the motor around the operating point are compared, considering demagnetization of the phases. In each of the tests, both models are stabilized at a speed of 2000rpm for 2 seconds and then a step is applied to the voltage input to vary the speed to 1000 , 1500,2500 and 3000rpm for 2 seconds and the behavior of the speed and torque responses is observed. Fig. 8 and 15. In each of the tests, the average values of speed and torque obtained in the two models were measured and the percentage error was calculated. The data collected is summarized in the table I.

TABLE I. ERROR AROUND THE OPERATING POINT

\begin{tabular}{|c|c|c|}
\hline $\begin{array}{c}\text { Operating speed } \\
(\mathbf{r p m})\end{array}$ & Speed error $(\boldsymbol{\%})$ & Torque error $(\boldsymbol{\%})$ \\
\hline 1000 & 8.4 & 2.8 \\
\hline 1500 & 2.7 & 0.4 \\
\hline 2500 & 1.5 & 0.8 \\
\hline 3000 & 2.7 & 1.2 \\
\hline
\end{tabular}

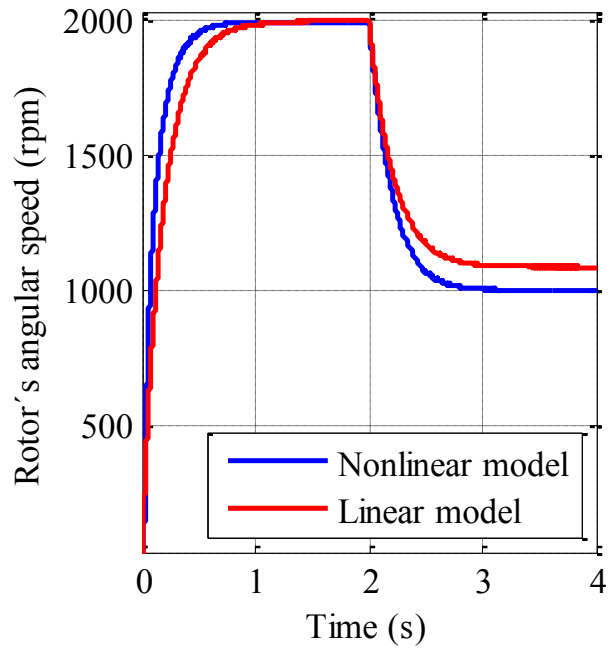

Fig. 8. Rotor's angular speed response, with step at $1000 \mathrm{rpm}$. 


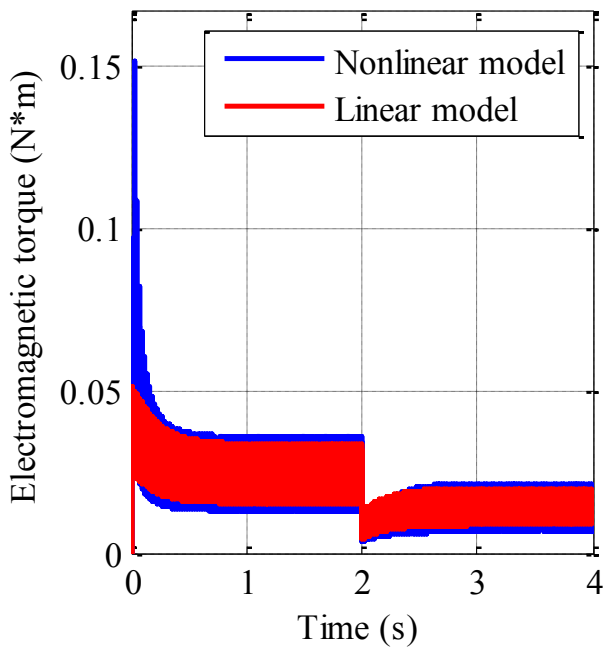

Fig. 9. Electromagnetic torque response, with step at $1000 \mathrm{rpm}$.

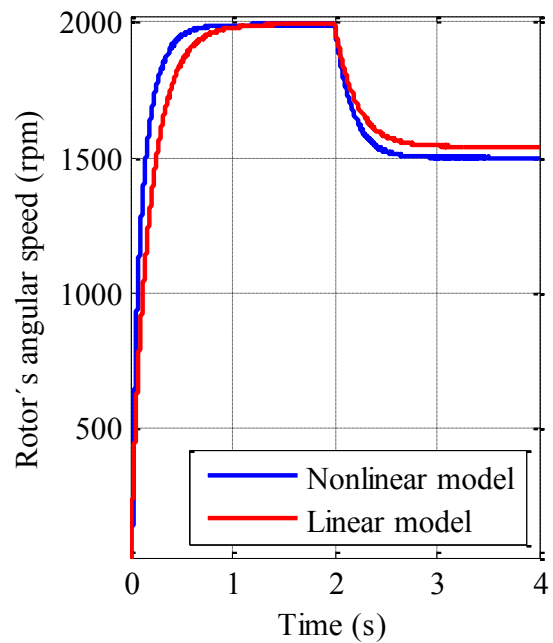

Fig. 10. Rotor's angular speed response, with step at $1500 \mathrm{rpm}$.

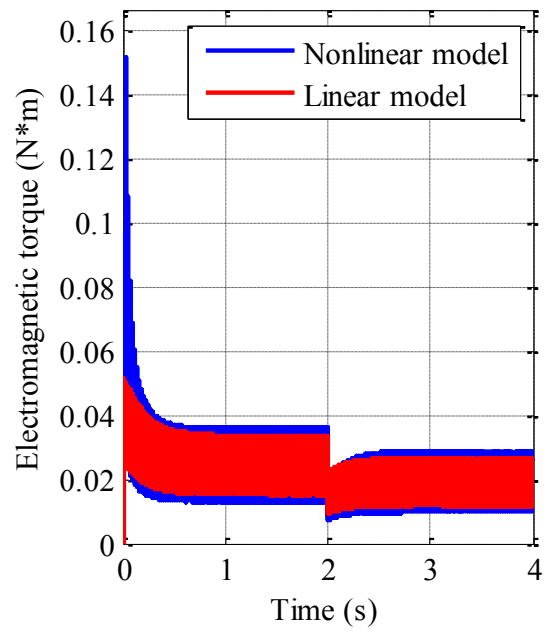

Fig. 11. Electromagnetic torque response, with step at $1500 \mathrm{rpm}$.

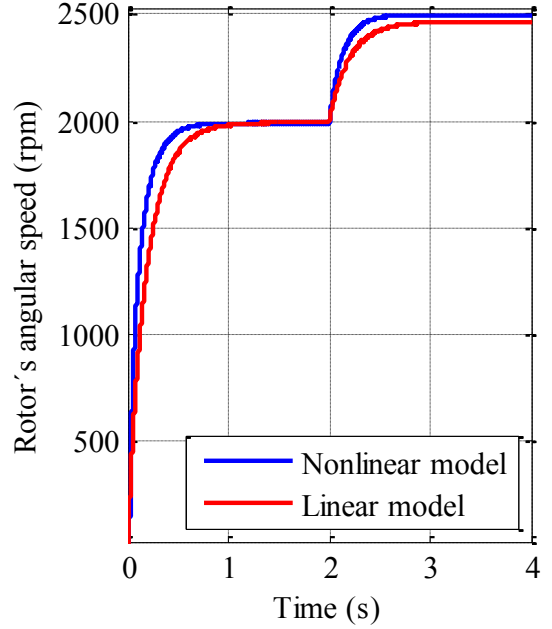

Fig. 12. Rotor's angular speed response, with step at $2500 \mathrm{rpm}$.

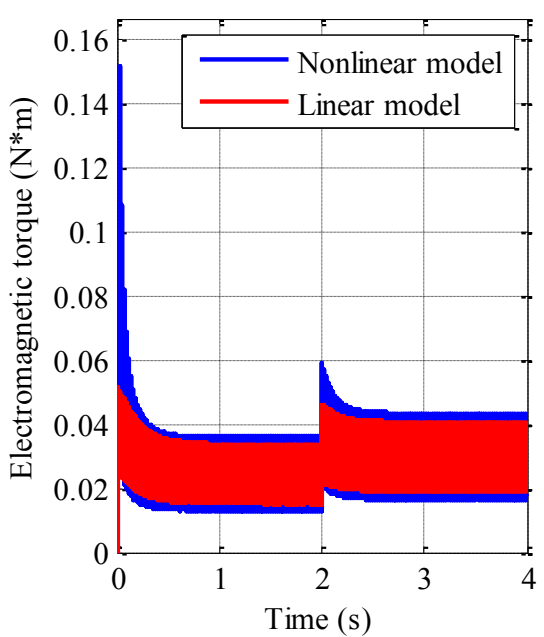

Fig. 13. Electromagnetic torque response, with step at $2500 \mathrm{rpm}$

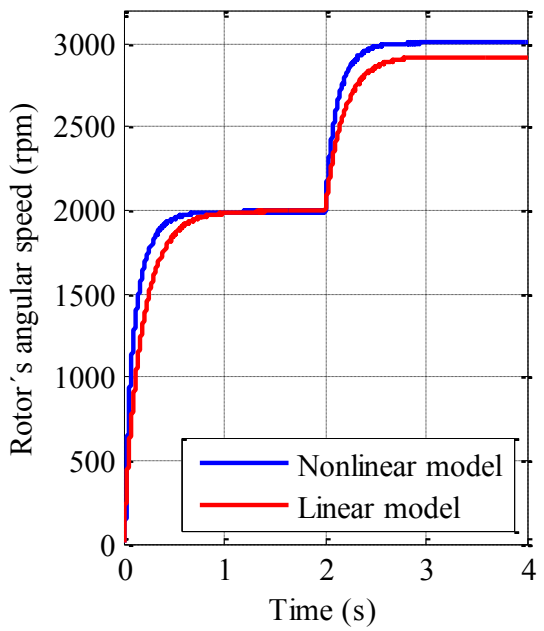

Fig. 14. Rotor's angular speed response, with step at $3000 \mathrm{rpm}$. 


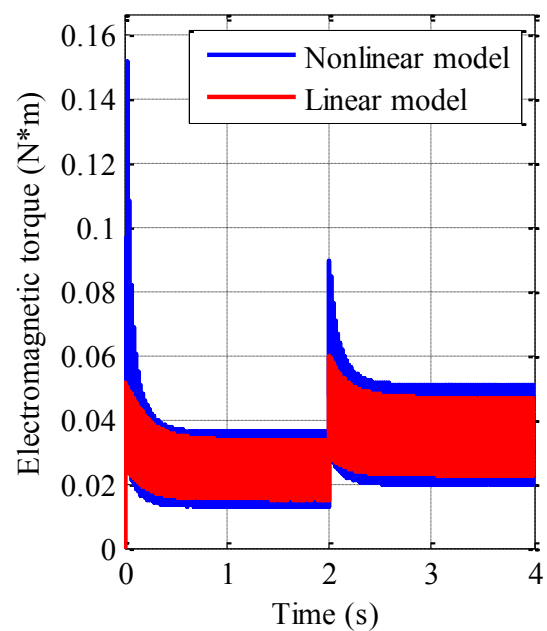

Fig. 15. Electromagnetic torque response, with step at $3000 \mathrm{rpm}$.

\section{Simulation with sinusoidal input}

In order to observe the behavior of the models to oscillatory variations, both models were simulated as in the previous experiment, considering demagnetization. For 2 seconds it is operated at 2000rpm and then a sinusoidal signal is applied with different frequencies, passing through $0.25,0.5,0.75$ and 1 $\mathrm{rad} / \mathrm{s}$ and with an amplitude that varies the operating point from 700 to $3000 \mathrm{rpm}$, the speed and torque responses for a frequency of $0.75 \mathrm{rad} / \mathrm{s}$ are shown in Fig. 16 and 17.

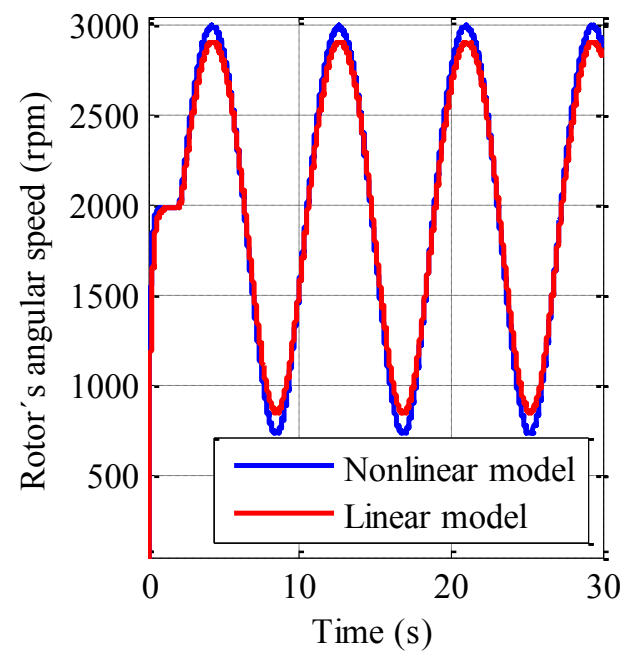

Fig. 16. Rotor's angular speed response, from 700 to $3000 \mathrm{rpm}$ at $0.75 \mathrm{rad} / \mathrm{s}$.

\section{DISCUSSION OF RESULTS}

From the first experiment, we can see how the linear model fails to reproduce the oscillations in the torque curves and consequently the same happens in the speed curves. This occurs, since, at the moment in which one phase is turned off and the next phase is turned on, the corresponding discharge and charge curves are compensated causing the total electromagnetic torque to be continuous. On the other hand, it

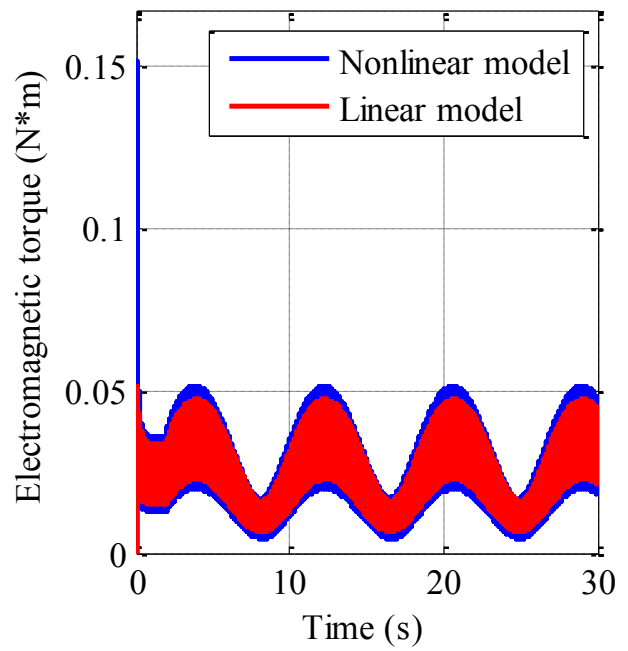

Fig. 17. Electromagnetic torque response, from 700 to $3000 \mathrm{rpm}$ at $0.75 \mathrm{rad} / \mathrm{s}$.

is observed how thanks to the demagnetization of the phases both models demonstrate a similar behavior in the speed and torque responses. This is due to the fact that the oscillating effect of the back EMF that differentiates both models is greatly reduced by demagnetization, since this causes the input voltage and voltage drop terms to be greater than the corresponding term. to the back EMF.

From the second experiment, we can see in table I, the error obtained, by varying the operating point of the motor in a range of $100 \%$ of the original operating point, remains below $8.4 \%$, obtaining a greater error at speeds below the operating point.

From the last experiment, it is observed how the linear model manages to replicate the behavior of the non-linear in the speed responses, maintaining a maximum error of $8.5 \%$ in the peaks of the oscillations, this without significantly changing the behavior when varying the frequency. Regarding torque, unlike what happened in the step tests, the responses obtained are more similar, since there are no abrupt changes in input voltage.

\section{CONCLUSION}

In this work, the modeling, analysis, linearization and simulation of a SRM 8/6 was presented considering Coulomb plus viscous friction and an ideal inverter circuit of bridge converter topology. From this work it was observed in the analysis part that, when trying to obtain an exact linearization of the system, to carry out a possible control using non-linear techniques, the result obtained is a linearization that would be useful only for position control, because it was necessary to change the output function. On the other hand, the transfer function of the linear model agrees with what is normally obtained in models of direct current motors, in which there are extremely separated poles between the electrical and mechanical subsystems; also, no zeros are obtained, just like expected from the zero dynamics of the nonlinear model. In addition, the linear model manages to reproduce the behavior of the non-linear model in a wide operating range, maintaining an error of less than $10 \%$. Therefore, it is feasible to design a 
classic controller based on the linear model, if phase demagnetization is considered.

Some recommendations for future work may be to compare the linear model with a non-linear model that considers the saturation phenomenon to observe the differences when the motor is subjected to saturation. It is also recommended to replace the ideal circuit with a circuit that has the characteristics of diodes and transistors to obtain a more complete model.

\section{References}

[1] K. Kiyota, T. Kakishima, and A. Chiba, "Comparison of test result and design stage prediction of switched reluctance motor competitive with $60-\mathrm{kw}$ rare-earth $\mathrm{pm}$ motor," IEEE Transactions on Industrial Electronics, vol. 61, no. 10, pp. 5712-5721, 2014.

[2] T. J. E. Miller, Electronic control of switched reluctance machines. Elsevier, 2001.

[3] R. Krishnan, Switched reluctance motor drives: modeling, simulation, analysis, design, and applications. CRC press, 2017.

[4] A. E. Fitzgerald, C. Kingsley, S. D. Umans, and B. James, Electric machinery. McGraw-Hill New York, 2003, vol. 5.

[5] X. Wang, R. Palka, and M. Wardach, "Nonlinear digital simulation models of switched reluctance motor drive," Energies, vol. 13, no. 24, p. 6715, 2020.

[6] K. Aiso and K. Akatsu, "High speed srm using vector control for electric vehicle," CES Transactions on Electrical Machines and Systems, vol. 4, no. 1, pp. 61-68, 2020.

[7] C. Li, G. Wang, J. Liu, Y. Li, and Y. Fan, "A novel method for modeling the electromagnetic characteristics of switched reluctance motors," Applied Sciences, vol. 8, no. 4, p. 537, 2018.

[8] P. Azer, S. Nalakath, B. Howey, B. Bilgin, and A. Emadi, "Dynamic vector modeling of three-phase mutually coupled switched reluctance machines with single dqquadrant lookup tables," IEEE Open Journal of the Industrial Electronics Society, vol. 1, pp. 271-283, 2020.

[9] I. S. Sarwar, "Modelling and motion control of bldc motor for pan tilt platform." WSEAS Transactions on Systems and Control, vol. 16, pp. 183-193, 2021.

[10] M. Tariq, T. Bhattacharya, N. Varshney, and D. Rajapan, "Fast response antiwindup pi speed controller of brushless dc motor drive: Modeling, simulation and implementation on dsp," Journal of electrical systems and information technology, vol. 3, no. 1, pp. 1-13, 2016.

[11] C. Xiang, X. Wang, Y. Ma, and B. Xu, "Practical modeling and comprehensive system identification of a bldc motor," Mathematical Problems in Engineering, vol. 2015, 2015.

[12] H. K. Khalil and J. W. Grizzle, Nonlinear systems. Prentice hall Upper Saddle River, NJ, 2002, vol. 3.

[13] H. Olsson, K. J. Åström, C. C. De Wit, M. Gäfvert, and P. Lischinsky, "Friction models and friction compensation," Eur. J. Control, vol. 4, no. 3, pp. 176-195, 1998.

[14]A. De la Guerra, "Observabilidad de motores de reluctancia conmutada," Master's thesis, Universidad Nacional Autónoma de México, Facultad de ingeniería, 2011.
[15]F. Khorrami, P. Krishnamurthy, and H. Melkote, Modeling and adaptive nonlinear control of electric motors. Springer Science \& Business Media, 2003.

[16] A. Isidori, E. Sontag, and M. Thoma, Nonlinear control systems. Springer, 1995, vol. 3.

[17] J. U. Liceaga-Castro, I. I. Siller-Alcalá, J. Jaimes-Ponce, R. A. Alcántara-Ramírez, and E. Arévalo Zamudio, "Identification and real time speed control of a series dc motor," Mathematical Problems in Engineering, vol. 2017, 2017.

[18] J. Jimenez-Gonzalez, F. Gonzalez-Montañez, V. M. Jimenez-Mondragon, J. U. Liceaga-Castro, R. EscarelaPerez, and J. C. Olivares-Galvan, "Parameter identification of bldc motor using electromechanical tests and recursive least-squares algorithm: Experimental validation," in Actuators, vol. 10, no. 7. Multidisciplinary Digital Publishing Institute, 2021, p. 143.

[19] C. A. Pérez-Gómez, J. U. Liceaga-Castro, and I. I. SillerAlcalá, "Comparative study between classical controllers and inverse dead zone control for position control of a permanent magnet dc motor with dead zone," in TRANSACTIONS on POWER SYSTEMS, vol. 15. WSEAS, 2020

[20] W. Piotr, Dynamics and control of electrical drives. Springer Science \& Business Media, 2011.

\section{Contribution of individual authors to the creation of a scientific article (ghostwriting policy)}

Jesús D. González-San Román programmed the models, carried out the simulations and contributed to the theoretical analysis.

Jesús U. Liceaga-Castro and Irma I. Siller-Alcalá contributed to the theoretical analysis.

Eduardo Campero-Littlewood carried out the electrical motor's analysis.

\section{Sources of funding for research presented in a scientific article or scientific article itself}

The project was funded by the UAM-Azc and by CONACYT scholarship grant to Jesús D. González-San Román

\section{Creative Commons Attribution License $\mathbf{4 . 0}$ (Attribution 4.0 International , CC BY 4.0)}

This article is published under the terms of the Creative Commons Attribution License 4.0 https://creativecommons.org/licenses/by/4.0/deed.en_ $\underline{\text { US }}$ 